\title{
A Quantum Linearity Test for Robustly Verifying Entanglement
}

\author{
Anand Natarajan \\ Center for Theoretical Physics, Massachusetts Institute of \\ Technology \\ Cambridge, Massachusetts, USA \\ anandn@mit.edu
}

\begin{abstract}
We introduce a simple two-player test which certifies that the players apply tensor products of Pauli $\sigma_{X}$ and $\sigma_{Z}$ observables on the tensor product of $n$ EPR pairs. The test has constant robustness: any strategy achieving success probability within an additive $\epsilon$ of the optimal must be poly $(\epsilon)$-close, in the appropriate distance measure, to the honest $n$-qubit strategy. The test involves $2 n$-bit questions and 2 -bit answers. The key technical ingredient is a quantum version of the classical linearity test of Blum, Luby, and Rubinfeld.

As applications of our result we give (i) the first robust selftest for $n$ EPR pairs; (ii) a quantum multiprover interactive proof system for the local Hamiltonian problem with a constant number of provers and classical questions and answers, and a constant completeness-soundness gap independent of system size; (iii) a robust protocol for verifiable delegated quantum computation with a constant number of quantum polynomial-time provers sharing entanglement.
\end{abstract}

\section{CCS CONCEPTS}

- Theory of computation $\rightarrow$ Quantum complexity theory; Interactive proof systems; Complexity classes;

\section{KEYWORDS}

Quantum Interactive Proofs, Self-Testing, Nonlocal Games, Quantum PCP Conjecture

\section{ACM Reference format:}

Anand Natarajan and Thomas Vidick. 2017. A Quantum Linearity Test for Robustly Verifying Entanglement. In Proceedings of 49th Annual ACM SIGACT Symposium on the Theory of Computing, Montreal, Canada, fune 2017 (STOC'17), 13 pages.

DOI: $10.1145 / 3055399.3055468$

\section{INTRODUCTION}

Quantum non-local games lie at the intersection of several areas of quantum information. They provide a natural approach to deviceindependent certification or self-testing of unknown quantum states. Device-independent certification has applications to quantum cryptography, from quantum key distribution $[22,30]$ to delegated computation $[10,28]$. The key idea behind these applications is that

Permission to make digital or hard copies of all or part of this work for personal or classroom use is granted without fee provided that copies are not made or distributed for profit or commercial advantage and that copies bear this notice and the full citation on the first page. Copyrights for components of this work owned by others than ACM must be honored. Abstracting with credit is permitted. To copy otherwise, or republish, to post on servers or to redistribute to lists, requires prior specific permission and/or a fee. Request permissions from permissions@acm.org.

STOC'17, Montreal, Canada

(c) 2017 ACM. 978-1-4503-4528-6/17/06 ...\$15.00

DOI: $10.1145 / 3055399.3055468$

\author{
Thomas Vidick \\ Department of Computing and Mathematical Sciences, \\ California Institute of Technology \\ Pasadena, California, USA \\ vidick@cms.caltech.edu
}

certain nonlocal games, such as the CHSH game [4], provide natural statistical tests that can be used to certify that an arbitrary quantum device implements a certain "strategy" specified by local measurements on an entangled state (e.g. an EPR pair). In complexity theory, non-local games are closely connected to the study of the class MIP* of languages having multiprover interactive proof systems with quantum entangled provers.

At the heart of many applications of non-local games is the problem of self-testing, which amounts to devising games in which success can be used to certify that the players share a particular entangled state. A common weakness of all existing self-testing results is that their performance scales poorly with the number of qubits of the state that is being tested. Given a self-test, define (somewhat informally) its robustness as the largest $\epsilon=\epsilon(\delta)$ such that a success probability at least $\omega_{\mathrm{opt}}^{*}-\epsilon$ in the test certifies the target state up to error (in trace distance and up to local isometries) at most $\delta$, where $\omega_{\mathrm{opt}}^{*}$ is the success probability achieved by an ideal strategy. All previously known tests for $n$-qubit states required $\epsilon \ll \operatorname{poly}(\delta, 1 / n)$. Aside from rendering these tests too inefficient to practically implement, this polynomial dependence on $n$ is an impediment to the strongest potential applications of non-local games in cryptography and complexity theory, including the problem of delegated computation and the quantum PCP conjecture.

Our main result is a form of robust self-test for any state that can be characterized via expectation values of tensor products of standard Pauli $\sigma_{X}$ or $\sigma_{Z}$ observables. (This includes a tensor product of $n$ EPR pairs; see below.)

Theorem 1.1 (SIMPLIFIED $^{1}$ ). Let $\mathcal{P}$ be a set of $n$-qubit observables, each of which is a tensor product of single-qubit Pauli $\sigma_{X}, \sigma_{Z}$ or $\pm I$, and $\lambda_{\max }=\left\|\mathbf{E}_{P \sim \mathcal{P}}[P]\right\|$. For any $\eta \geq 0$ there exists $a p=p(\eta)=$ $\Theta\left(\eta^{c}\right)$, where $0<c<1$ is a universal constant, and a 7-player nonlocal game with $O(n)$-bit questions and $O(1)$-bit answers such that

$$
\omega_{\mathrm{opt}}^{*}=\frac{1}{2}+p \lambda_{\max } \pm \eta
$$

We view the theorem as a robust self-test in the following sense. Suppose a many-qubit state $|\psi\rangle$ can be characterized as the leading eigenvector of an operator $O=\mathbf{E}_{P \sim \mathcal{P}}[P]$ obtained as the average of $n$-qubit Pauli operators, with associated eigenvalue $\lambda_{\max } \in[-1,1]$. For example, if $\mathcal{P}$ is the uniform distribution over $\left\{\sigma_{X} \otimes \sigma_{X}, \sigma_{Z} \otimes\right.$ $\left.\sigma_{Z}\right\}^{\otimes n}$ then $\lambda_{\max }=1$ and the leading eigenvector is the tensor product of $n$ EPR pairs. More generally, if $H$ is a local Hamiltonian with $m$ local $X Z$ terms (i.e. tensor products of single-qubit Pauli $\sigma_{X}$ and $\sigma_{Z}$ observables) we can take $\mathcal{P}$ to be $\mathbb{I}$ with probability $1 / 2$ and the negation of a random term of $H$ with probability $1 / 2$. Then

\footnotetext{
${ }^{1}$ The complete statement of the theorem says much more, and provides a characterization of near-optimal strategies.
} 
$\lambda_{\max }=\frac{1}{2}-\frac{1}{2 m} \lambda_{\min }(H)$ and the leading eigenvector is a ground state of $H$.

Theorem 1.1 provides a nonlocal game such that the optimal success probability in the game is directly related to $\lambda_{\max }$, thereby providing a test distinguishing between small and large $\lambda_{\max }$. In fact the complete statement of the theorem (see Theorem 5.2 in Section 5) says much more. In particular, we provide a complete characterization (up to local isometries) of strategies achieving a success probability at least $\omega_{\text {opt }}^{*}-\epsilon$, for $\epsilon$ sufficiently small but independent of $n$, showing that such strategies must be based on a particular encoding (based on a simple, fixed error-correcting code) of an eigenstate associated to $\lambda_{\max }$.

\subsection{Applications}

Before giving an overview of the proof of the theorem we discuss some consequences of the theorem that help underscore its generality.

Hamiltonian Complexity. A first consequence of Theorem 1.1 is that the ground state energy of a local Hamiltonian can be certified via a non-local game with questions of polynomial length and constant-length answers.

Corollary 1.2. Let $H$ be an $n$-qubit Hamiltonian that can be expressed as a weighted sum, with real coefficients, of tensor products of $\sigma_{X}$ and $\sigma_{Z}$ operators on a subset of the qubits, and normalize $H$ such that $\|H\| \leq 1$. Suppose it is given that $\lambda_{\min }(H) \leq a$ or $\lambda_{\min }(H) \geq b$ for some $0 \leq a<b \leq 1$. There exists a one-round interactive proof system between a classical polynomial-time verifier and 7 entangled provers where the verifier's (classical) questions are $O(n /(b-a))$ bits long, the provers' (classical) answers are $O(1)$ bits each, and the maximum probability that the verifier accepts is

$$
\begin{aligned}
& \lambda_{\min } \leq a \Longrightarrow \omega_{\mathrm{opt}}^{*} \geq p_{\mathrm{c}}:=\frac{1}{2}+2 \eta_{0}, \\
& \lambda_{\min } \geq b \Longrightarrow \omega_{\mathrm{opt}}^{*} \leq p_{\mathrm{s}}:=\frac{1}{2}+\eta_{0},
\end{aligned}
$$

where $\eta_{0}>0$ is a small (universal) constant.

Since the class of Hamiltonians considered in Corollary 1.2 is QMA-complete [8], the corollary can be viewed as a quantum analogue of the (games variant of the) exponentially long PCP based on the linearity test of Blum, Luby and Rubinfeld [2]. Indeed, observe that the game constructed in the corollary has an efficient verifier, polynomial-length questions, and a constant completenesssoundness gap $\eta_{0}$ as soon as the original promise on the ground state energy for the Hamiltonian exhibits an inverse-polynomial completeness-soundness gap. The derivation of Corollary 1.2 from Theorem 1.1 involves a step of gap amplification via tensoring, and relies on the fact that Theorem 1.1 allows any $X Z$-Hamiltonian with no requirement on locality.

A similar result to Corollary 1.2 was obtained by Ji [15], and we build on Ji's techniques. The results are incomparable: on the one hand, the question size in our protocol is much larger $(\operatorname{poly}(n)$ bits instead of $O(\log n)$ for [15]); on the other hand, the dependence of the verifier's acceptance probability on the ground state energy is much better, as in [15] the completeness-soundness gap remains inverse polynomial.
An Exponential Quantum PCP.. The expert reader may already have noted that the complexity-theoretic formulation of Corollary 1.2 described above already follows from known results in quantum complexity. Indeed, recall that the class QMA is in PSPACE, and that single-round multiprover interactive proof systems for PSPACE (and even NEXP) follow from the results in [14, 31]. Another possible proof approach for the same result could be obtained by repeating the protocol in [15] a polynomial number of times; provided there existed an appropriate parallel repetition theorem this would amplify the soundness to a constant (although the answer length would now be polynomial). In fact, based on a recent result by Ji [16] it seems likely that both approaches, based either on our results or parallel repetition of [16], could lead to an exponential "quantum-games" PCP for all languages in NEXP (instead of just QMA). Even though in purely complexity-theoretic terms the result would still not be new, we believe that the techniques developed to obtain it show good promise for further extensions to Hamiltonian complexity.

Indeed our protocol has some advantages over the generic sequence of known reductions described above. One is efficiency: in our protocol the provers merely need access to a ground state of the given local Hamiltonian and the ability to perform constant-depth quantum circuits. It is this property that enables our application to delegated quantum computing (see below). Answers in our protocol are a constant number of bits; the reductions mentioned above would require soundness amplification via parallel repetition, which would lead to answers of (at least) linear length.

Even though they may not provide the most immediately compelling application of Theorem 1.1, the consequences of Corollary 1.2 to complexity theory tie our results to one of their primary motivations, the quantum PCP conjecture. Broadly speaking, the quantum PCP research program is concerned with finding a robust analog of the Cook-Levin theorem for the class QMA. The "games variant" of this conjecture states that estimating the optimal winning probability of entangled players in a multiplayer nonlocal game, up to an additive constant, is QMA-hard. In other words, that there exists an MIP* protocol for QMA with $O(\log (n))$-bit messages and constant completeness-soundness gap. The best progress to date in this direction is the work of Ji [15], which gives a five-prover one-round MIP* protocol with $O(\log (n))$-bit messages for the local Hamiltonian problem such that the verifier's maximum acceptance probability is $a-b \lambda_{\min }(H) n^{-c}$ for positive constants $a, b, c$. This falls short of the games PCP conjecture in that the completenesssoundness gap is inverse polynomial in $n$, rather than constant. ${ }^{2}$

Our results suggest an approach to the problem from a different angle: we provide a "gap preserving" protocol, in the sense that the completeness-soundness gap is a polynomial function of the underlying promise gap of the Hamiltonian, but independent of the system size $n$. However, this occurs at the cost of much longer messages - polynomial instead of logarithmic.

Dimension Witnesses. Consider the operator $O=\left(\frac{1}{2}\left(\sigma^{X} \otimes \sigma^{X}+\right.\right.$ $\left.\left.\sigma^{Z} \otimes \sigma^{Z}\right)\right)^{\otimes n}$. This operator has largest eigenvalue 1 with associated eigenvector $|\mathrm{EPR}\rangle^{\otimes n}$, where $|\mathrm{EPR}\rangle=\frac{1}{\sqrt{2}}|00\rangle+\frac{1}{\sqrt{2}}|11\rangle$. In this case

${ }^{2}$ Here again we point the interested reader to the recent [16], which obtains a protocol with similar parameters, involving 8 provers, for all languages in NEXP. 
the proof of Theorem 1.1 allows us to obtain the following robust self-test for $|\mathrm{EPR}\rangle^{\otimes n}$ :

COROLlARy 1.3. For any integer $n$ there is a two-player game with $O(n)$-bit questions and $O(1)$-bit answers such that (i) there is a strategy with optimal winning probability $0<\omega^{*} \leq 1$ that uses $|\mathrm{EPR}\rangle^{\otimes n}$ as entangled state; (ii) for any $\epsilon>0$, any strategy with success probability at least $\omega^{*}-\epsilon$ must be based on an entangled state which is (up to local isometries) within distance $\delta=\operatorname{poly}(\epsilon)$ of $|\mathrm{EPR}\rangle^{\otimes n}$.

The game whose properties are summarized in Corollary 1.3 is based on the CHSH game. By using the Magic Square game instead, it is possible to devise a test with perfect completeness, $\omega^{*}=1$, which can be achieved using an honest strategy based on the use of $(n+1)$ EPR pairs.

To the best of our knowledge, all prior self-tests for any family of states had a robustness guarantee going to 0 inverse polynomially fast with the number of qubits tested (see Section 1.3 below for a more thorough comparison with related works).

Delegated Computation. It was noticed in [10] that an interactive proof system for the local Hamiltonian problem can also be used for delegated quantum computation with so-called post-hoc verification. The key idea is to use the Feynman-Kitaev construction to produce a Hamiltonian encoding the desired computation; measuring the ground energy of this Hamiltonian reveals whether the computation accepts or rejects. Following the same connection, we are able to give a post-hoc verifiable delegated computation scheme with a purely classical verifier and a constant number of provers. The provers only need the power of BQP. The scheme has a constant completeness-soundness gap independent of the size of the circuit to be computed, unlike the scheme of [10] and the classical scheme of [28], which both have inverse-polynomial gaps. However, unlike the scheme of [28], our protocol is not blind: the verifier must reveal the entire circuit to be computed to all the provers before the verification process starts. We refer to the full version [24] for more details on this application.

\subsection{Proof Overview}

The proof of Theorem 1.1 builds on ideas from complexity theory and quantum information. We draw inspiration from classical ideas in the closely related areas of probabilistically checkable proofs, locally testable codes, and property testing. The link between these areas and quantum self-testing is the idea of verifying a global property of an unknown object using only limited measurements. The two most important components of the proof are a "locally verifiable" encoding of arbitrary $n$-qubit quantum states [11], and a quantum analogue of the linearity test of Blum et al. [2]. Since the second component is the more novel we explain it first.

Linearity Testing of Quantum Observables. The simplest instantiation of the classical PCP theorem relies on the Hadamard code to robustly encode an $n$-bit string (e.g. an assignment to an instance of 3-SAT). Under this code, a string $u \in\{0,1\}^{n}$ is encoded as the $2^{n}$-bit long truth table of the function $f_{u}:\{0,1\}^{n} \rightarrow\{-1,1\}$ given by $f_{u}(x)=(-1)^{u \cdot x}$, where $\cdot$ is the bitwise inner product. The function $f_{u}(x)$ is said to be linear, since $f_{u}(x+y)=f_{u}(x) f_{u}(y)$. The key property of the Hadamard code which makes it useful in this context is that it is locally testable. A local test is given by the BLR linearity test: given query access to a function $f:\{0,1\}^{n} \rightarrow\{-1,1\}$, by checking that $f(x+y)=f(x) f(y)$ at randomly chosen $x, y$ the test certifies that any $f$ that is accepted with probability at least $1-\epsilon$ has the form $f \approx_{\epsilon} f_{u}$ for some $u \in\{0,1\}^{n}$, where $f_{u}: x \mapsto(-1)^{u \cdot x}$ and $\approx_{\epsilon}$ designates equality on an $(1-O(\epsilon))$ fraction of inputs.

Here is a "quantum" reformulation of this test as a nonlocal game: instead of querying an oracle for $f$ at three aligned points, play a three-player nonlocal game where each player is asked for the value at a point. This test is sound even if the players share an entangled quantum state [14], but success in the test does not certify quantum behavior: the players could win with certainty just by sharing a description of a classical linear function $f_{u}$. (Indeed, the main point of the analysis in [14] is precisely to ensure that provers sharing entanglement have no more freedom than to use it as shared randomness in selecting $u$.)

In contrast, we seek an extension of the test which certifies a very specific type of quantum behavior, that could not be emulated by classical means alone: specifically, that the observable $O_{x}$ measured by a player upon receiving question $x$ itself is (up to a change of basis, and in the appropriate "state-dependent" norm) close to $\otimes_{i} \sigma_{X}^{x_{i}}$. We give a test which achieves this. The test performs a combination of a linearity test in the $X$-basis and a linearity test in the $Z$-basis; an "anticommutation game" (which can be taken to be a version of the CHSH or Magic Square games) is used to constrain how the results of the two linearity tests relate to each other.

Theorem 1.4 (PAuli Braiding test, Informal). There exists $a$ two-player nonlocal game, based on the combination of $(i)$ a linearity test in the $X$ basis (questions $x \in\{0,1\}^{n}$ ); (ii) a linearity test in the $Z$ basis (questions $z \in\{0,1\}^{n}$ ); (iii) an "anticommutation game" (based on e.g. the CHSH or Magic Square games) designed to test for generalized anti-commutation relations (questions $(x, z) \in\{0,1\}^{2 n}$ ), such that any strategy that has success probability $\omega_{\mathrm{opt}}^{*}-\epsilon$ for some $\epsilon>0$ (where $0<\omega_{\mathrm{opt}}^{*} \leq 1$ depends on the anticommutation game used) must be based on observables $A(x), A(z), A(x, z)$ and an entangled state $|\psi\rangle_{A B}$ such that up to local isometries

$$
A(x) \approx_{\delta} \otimes_{i} \sigma_{X}^{x_{i}}, A(z) \approx_{\delta} \otimes_{i} \sigma_{Z}^{z_{i}}, \text { and }|\psi\rangle_{A B} \approx_{\delta}|\mathrm{EPR}\rangle_{A B}^{\otimes n},
$$

where $\delta=\operatorname{poly}(\epsilon)$. Moreover, there exists a strategy for the provers which succeeds with probability $\omega_{\mathrm{opt}}^{*}$ and uses $n$ EPR pairs.

Neither the linearity test nor the anticommutation test alone would be sufficient to achieve the conclusion: as noted above, the linearity test can be passed even by classical provers, and our anticommutation test can be fooled if the provers share just one EPR pair. Rather, it is the guarantees provided by these tests together that enable us to create a tensor-product structure in the provers' Hilbert space.

To gain intuition on the test one may think of it in the following way. A standard approach to self-testing $n$ EPR pairs is to fix a decomposition of the Hilbert space as

$$
\mathcal{H}=\mathbb{C}^{2^{n}} \approx \mathbb{C}^{2} \otimes \cdots \otimes \mathbb{C}^{2},
$$

and perform the CHSH (or Magic Square) test "in parallel", on each copy of $\mathbb{C}^{2}$. To the best of our knowledge such tests only leads to robustness bounds with a polynomial dependence in $n$. In contrast the test on which Theorem 1.4 is based relies on the observation 
that the decomposition (1) need not be rigidly fixed a priori; indeed there are many bases in which such decomposition of $\mathbb{C}^{2^{n}}$ in tensor factors can be performed. In particular, any pair of anti-commuting observables on $\mathcal{H}$ suffices to specify a copy of $\mathbb{C}^{2}$, on which a $\mathrm{CHSH}$ test can in principle be performed (here we crucially rely on rotation invariance of the $2^{n}$-dimensional maximally entangled state). Our test leverages this observation by performing a CHSH test for each possible pair of Pauli operators $\left(\sigma_{X}(a), \sigma_{Z}(b)\right)$, where $a, b \in\{0,1\}^{n}$ are such that $a \cdot b=1$. Each of these tests amounts to identifying a copy of $\mathbb{C}^{2}$ and performing the CHSH test on it. Contrary to the parallel-repeated CHSH test these copies are not independent, and this is what makes our test much more robust.

Encoding Quantum States. The second component of the proof of Theorem 1.1 is a procedure, first introduced in $[11,15]$, for encoding an $n$-qubit quantum state in a constant number of $n$-qubit shares such that certain properties of the encoded state (such as expectation values of local Pauli observables) can be verified through a classical interaction with provers each holding one of the shares. This is akin to how the "games" variant of the classical PCP theorem is derived from the "proof-checking" variant: while in the classical setting a proof can be directly shared across multiple provers, in the quantum setting we use a form of secret-sharing code that allows for distributing quantum information.

This procedure is efficient in that the total number of tests that can be performed (equivalently, the number of questions) is polynomial in $n$. However, the test in $[11,15]$ is not robust, and is only able to provide meaningful results for values of $\epsilon$ that scale inverse-polynomially with $n$. By extending the Pauli braiding test, Theorem 1.4, to the stabilizer framework of [15] we obtain a procedure which is meaningful for constant $\epsilon$. The drawback is that the provers may now be asked to measure all their qubits, and questions have length linear in $n$; however the total effort required of the classical verifier (and of provers given access to the state) remains polynomial in the size of the instance.

\subsection{Related Work}

We build on a number of previous works in quantum information and complexity theory. Motivation for the problem we consider goes back to a question of Aharonov and Ben-Or (personal communication, 2013), who asked how a quantum generalization of the exponential classical PCP could look like if it was not derived through the "circuitous route" obtained as the compilation of known but complex results from the theory of classical and quantum interactive proof systems (as described earlier). In this respect we point to [1, Section 5] for a very different approach to the same question based on a "quantum take" on the arithmetization technique.

More directly, our work builds on the aforementioned works [11, 15] initiating the study of entangled-prover interactive proof systems for the local Hamiltonian problem. The idea of using a distributed encoding of the ground state in order to obtain a multiprover interactive proof system for the ground state energy is introduced in [11]. In that work the protocol required the provers to return qubits; the possibility for making the protocol purely classical was uncovered by Ji [15]. Our use of stabilizer codes, and the stabilizer test which forms part of our protocol, originate in his work.
In addition we borrow from ideas introduced in the study of quantum multiprover interactive proofs with entangled provers [5, 17], and especially the three-prover linearity test of [14] and the use of oracularization from [13] to make it into a two-prover test.

Our results are related to work in quantum self-testing, in particular testing EPR pairs [20] and more general entangled states [18]. A sequence of results has established that the presence of $n$ EPR pairs between two provers can be certified via a protocol using queries and answers of length polynomial in $n$, with inverse-polynomial completeness-soundness gap. This was first achieved by [28] for a test based on serial repetition of the $\mathrm{CHSH}$ game, and subsequently by [19] for a single-round test based on CHSH, by [26] for an XOR game based on $\mathrm{CHSH}$, and by [7] and [6] independently for the parallel-repeated Magic Square game. Viewed in the context of these results our work is the only one to provide a test whose robustness does not depend on the number of EPR pairs being tested. The reason this can be achieved is the linearity test(s) performed as part of the Pauli braiding test, which we see as a major innovation of our work

\subsection{Open Questions and Future Directions}

In our opinion the most important direction for future work is to improve the efficiency of the Pauli braiding test in terms of the number of questions required. Can the test be derandomized, to questions of sub-linear, or even logarithmic, length? Such a result would establish the main step left towards proving the games variant of the quantum PCP conjecture. Instead of directly derandomizing the current test, can it be made more robust, perhaps using some of the ideas based on low-degree polynomial encodings that are key to the classical PCP theorem?

Aside from this challenging problem, there are several open questions that we find interesting and may be more approachable.

(1) In the classical PCP setting, the Hadamard code and the BLR linearity test can be used for alphabet reduction: converting a PCP or MIP protocol with large answer alphabet into one with a binary alphabet. This is a key step in Dinur's proof of the PCP theorem [9]. Can the linearity test also be used for alphabet reduction of MIP* protocols? The difficulty is to preserve completeness; if the optimal honest strategy uses a maximally entangled state then the adaptation should be straightforward, but if not it may be more challenging - perhaps ideas similar to our protocol for ground states of $X Z$ Hamiltonians can be used.

(2) An obvious application for many EPR pairs is quantum key distribution (QKD). A major contribution of [28] was to show that the sequential self-test for many EPR pairs obtained in that paper could be leveraged into a scheme for quantum key distribution (QKD) that is secure in the device-independent (DI) model of security. We believe it should be possible to use the Pauli braiding test to develop a DIQKD protocol in which the interaction with the devices can be executed in parallel, but we leave this possibility for future work.

(3) The energy test can be viewed as a "device independent property test" for any property that can be expressed as the energy of a suitable Hamiltonian acting on a quantum state. 
Are there other device-independent property tests that can be formulated in our framework? It would be interesting to see which results from the survey of Montanaro and de Wolf on quantum property testing [23] can be generalized to the device-independent setting.

Organization of the Paper. In Section 2 we introduce some notation used throughout as well as basic definitions of stabilizer codes and local Hamiltonians. In Section 3, we establish an important technical component of our results, the linearity test and its quantum analysis. We expand this into a two-prover self-test for the Pauli group on $n$ qubits in Section 4, which forms the basis for our main result. In Section 5 we extend this test to handle more than two provers and show how it can be combined with an energy measurement test to devise a game for the local Hamiltonian problem.

\section{PRELIMINARIES}

We assume basic familiarity with quantum information but give all required definitions. We refer to the standard textbook [25] for additional background material.

\subsection{Quantum States and Measurements}

A $n$-qubit pure quantum state is represented by a unit vector $|\psi\rangle \in$ $\mathbb{C}^{2} \otimes \cdots \otimes \mathbb{C}^{2}=\left(\mathbb{C}^{2}\right)^{\otimes n} \approx \mathbb{C}^{2^{n}}$, where the ket notation $|\cdot\rangle$ is used to signify a column vector. A bra $\langle\psi|$ is used for the conjugatetranspose $\langle\psi|=| \psi\rangle^{\dagger}$, which is a row vector. We use $\||\psi\rangle \|^{2}=$ $|\langle\psi \mid \psi\rangle|$ to denote the Euclidean norm, where $\langle\psi \mid \phi\rangle$ is the skewHermitian inner product between vectors $|\phi\rangle$ and $|\psi\rangle$. A $n$-qubit mixed state is represented by a density matrix, a positive semidefinite matrix $\rho \in \mathbb{C}^{2^{n}} \times \mathbb{C}^{2^{n}}$ of trace 1 . The density matrix associated to $|\psi\rangle$ is the rank-1 projection $|\psi\rangle\langle\psi|$. We use $\mathrm{D}(\mathcal{H})$ to denote the set of all density matrices on $\mathcal{H}$.

For a matrix $X,\|X\|$ will refer to the operator norm, the largest singular value. When the Hilbert space can be decomposed as $\mathcal{H}=$ $\mathcal{H}_{A} \otimes \mathcal{H}_{B}$ for some $\mathcal{H}_{A}$ and $\mathcal{H}_{B}$, and $X$ is an operator on $\mathcal{H}_{A}$, we often write $X$ as well for the operator $X \otimes \mathbb{I}_{\mathcal{H}_{B}}$ on $\mathcal{H}$. It will always be clear from context which space an operator acts on. All Hilbert spaces considered in the paper are finite dimensional.

We use $\operatorname{Pos}(\mathcal{H})$ to denote the set of positive semidefinite operators on $\mathcal{H}$. A $n$-qubit measurement (also called POVM, for projective operator-valued measurement) with $k$ outcomes is specified by $k$ positive matrices $M=\left\{M_{1}, \ldots, M_{k}\right\} \subseteq \operatorname{Pos}\left(\mathbb{C}^{2^{n}}\right)$ such that $\sum_{i} M_{i}=\mathbb{I}$. The measurement is projective if each $M_{i}$ is a projector, i.e. $M_{i}^{2}=M_{i}$. The probability of obtaining the $i$-th outcome when measuring state $\rho$ with $M$ is $\operatorname{Tr}\left(M_{i} \rho\right)$. By Naimark's dilation theorem, any POVM can be simulated by a projective measurement acting on an enlarged state; that is, for every POVM $M=\left\{M_{i}\right\}_{i}$ acting on state $|\psi\rangle \in \mathcal{H}$ there exists a projective measurement $M^{\prime}=\left\{P_{i}\right\}_{i}$ and a state $|\psi\rangle \otimes|\phi\rangle \in \mathcal{H} \otimes \mathcal{H}_{\text {ancilla }}$ with the same outcome probabilities as $M$. Moreover, the post-measurement state after performing $M$ is the same as the reduced post-measurement state obtained after performing $M^{\prime}$ and tracing out the ancilla subsystem $\mathcal{H}_{\text {ancilla }}$.

An $n$-qubit observable is a Hermitian matrix $O \in \mathbb{C}^{2^{n}} \times \mathbb{C}^{2^{n}}$ that squares to identity. We use $\operatorname{Obs}(\mathcal{H})$ to denote the set of observables
Table 1: Stabilizer table for the 7-qubit Steane code

\begin{tabular}{l|ccccccc} 
& 1 & 2 & 3 & 4 & 5 & 6 & 7 \\
\hline Stabilizers & $I$ & $I$ & $I$ & $\sigma_{X}$ & $\sigma_{X}$ & $\sigma_{X}$ & $\sigma_{X}$ \\
& $I$ & $\sigma_{X}$ & $\sigma_{X}$ & $I$ & $I$ & $\sigma_{X}$ & $\sigma_{X}$ \\
& $\sigma_{X}$ & $I$ & $\sigma_{X}$ & $I$ & $\sigma_{X}$ & $I$ & $\sigma_{X}$ \\
& $I$ & $I$ & $I$ & $\sigma_{Z}$ & $\sigma_{Z}$ & $\sigma_{Z}$ & $\sigma_{Z}$ \\
& $I$ & $\sigma_{Z}$ & $\sigma_{Z}$ & $I$ & $I$ & $\sigma_{Z}$ & $\sigma_{Z}$ \\
\hline Logical X & $\sigma_{Z}$ & $I$ & $\sigma_{Z}$ & $I$ & $\sigma_{Z}$ & $I$ & $\sigma_{Z}$ \\
Logical Z & $\sigma_{Z}$ & $\sigma_{X}$ & $\sigma_{X}$ & $\sigma_{X}$ & $\sigma_{X}$ & $\sigma_{X}$ & $\sigma_{X}$ \\
& & & $\sigma_{Z}$ & $\sigma_{Z}$ & $\sigma_{Z}$ & $\sigma_{Z}$ & $\sigma_{Z}$
\end{tabular}

acting on $\mathcal{H} . O \in \operatorname{Obs}(\mathcal{H})$ is diagonalizable with eigenvalues \pm 1 , $O=P_{+}-P_{-}$, and $P=\left\{P_{+}, P_{-}\right\}$is a projective measurement. For any state $\rho, \operatorname{Tr}(O \rho)$ is the expectation of the \pm 1 outcome obtained when measuring $\rho$ with $P$. If $\rho=|\psi\rangle\langle\psi|$ we abbreviate this quantity, $\operatorname{Tr}(O \rho)=\operatorname{Tr}\left(P_{+} \rho\right)-\operatorname{Tr}\left(P_{-} \rho\right)=\langle\psi|O| \psi\rangle$ as $\langle P\rangle_{\psi}$.

A convenient orthogonal basis for the real vector space of $n$ qubit observables is given by the set $\left\{I, \sigma_{X}, \sigma_{Y}, \sigma_{Z}\right\}^{\otimes n}$ of tensor products of the four single-qubit Pauli observables

$$
I=\left(\begin{array}{ll}
1 & 0 \\
0 & 1
\end{array}\right), \sigma_{X}=\left(\begin{array}{ll}
0 & 1 \\
1 & 0
\end{array}\right), \sigma_{Y}=\left(\begin{array}{cc}
0 & -i \\
i & 0
\end{array}\right), \sigma_{Z}=\left(\begin{array}{cc}
1 & 0 \\
0 & -1
\end{array}\right)
$$

We call the eigenbasis of $\sigma_{X}$ (resp. $\sigma_{Z}$ ) the $X$-basis (resp. $Z$-basis). We often consider operators that are tensor products of just $I$ and $\sigma_{X}$, or just $I$ and $\sigma_{Z}$. We denote these by $\sigma_{X}(a), \sigma_{Z}(b)$, where the strings $a, b \in\{0,1\}^{n}$ indicate which qubits to apply the $\sigma_{X}$ or $\sigma_{Z}$ operators to: a 0 in position $i$ indicates an $I$ on qubit $i$, and a 1 indicates an $\sigma_{X}$ or $\sigma_{Z}$. We denote by

$$
|\mathrm{EPR}\rangle=\frac{1}{\sqrt{2}}|0\rangle|0\rangle+\frac{1}{\sqrt{2}}|1\rangle|1\rangle
$$

the unique state stabilized by both $\sigma_{X} \otimes \sigma_{X}$ and $\sigma_{Z} \otimes \sigma_{Z}$.

\subsection{Stabilizer Codes}

Stabilizer codes are the quantum analogue of linear codes. For an introduction to the theory of stabilizer codes we refer to [12]. We will only use very elementary properties of such codes.

The codes we consider are Calderbank-Shor-Steane (CSS) codes [3, 29 ] that arise from self-dual classical codes. For an $r$-qubit code the codespace, the vector space of all valid codewords, is the subspace of $\left(\mathbb{C}^{2}\right)^{\otimes r}$ that is the simultaneous +1 eigenspace of a set $\left\{S_{1}, \ldots, S_{k}\right\}$ of $r$-qubit pairwise commuting Pauli observables called the stabilizers of the code. The stabilizers form a group under multiplication. Unitary operations, such as a Pauli $X$ or $Z$ operators, on the logical qubit are implemented on the codespace by logical operators $X_{\text {logical }}$ and $Z_{\text {logical }}$. The smallest CSS code is Steane's 7-qubit code [29]. Table 1 lists a set of stabilizers that generate the stabilizer group of the code.

This class of codes satisfies certain properties which will be useful for us. Firstly, both the stabilizer generators and the logical operators can be written as tensor products of only $I, \sigma_{X}$, and $\sigma_{Z}$ operators - there are no $\sigma_{Y}$. This simplifies our protocol, allowing us to consider only two distinct basis settings. Secondly, every CSS code arising from a self-dual classical code has the following symmetry: for every index $i \in[r]$ there exists stabilizers $S_{X}, S_{Z}$ such that $S_{X}$ is a tensor product of only $\sigma_{X}$ and $I$ operators and 
has an $\sigma_{X}$ at position $i$, and $S_{Z}$ is equal to $S_{X}$ with all $\sigma_{X}$ operators replaced by $\sigma_{Z}$ operators.

These properties imply the following simple observation, which will be important for us. For every Pauli operator $P \in\left\{I, \sigma_{X}, \sigma_{Z}\right\}$ acting on the $i$-th qubit of the code there is a tensor product $\bar{P}$ of Paulis acting on the remaining $(r-1)$ qubits such that $P \otimes \bar{P}$ is a stabilizer operator on the whole state, and moreover each term in the tensor product is either identity or $P$. Indeed, the choice of $\bar{P}$ is not unique. Henceforth, we use the notion $\bar{P}$ to denote any such operator, unless otherwise specified.

\subsection{Local Hamiltonians}

A $n$-qubit local Hamiltonian is a Hermitian, positive semidefinite operator $H$ on $\left(\mathbb{C}^{2}\right)^{\otimes n}$ that can be decomposed as a sum $H=$ $\sum_{i=1}^{m} H_{i}$ with each $H_{i}$ is local, i.e. $H_{i}$ can be written as $H_{i}=I \otimes$ $\cdots I \otimes h_{i} \otimes I \otimes \cdots \otimes I$, where $h_{i}$ is a Hermitian operator on $\left(\mathbb{C}^{2}\right)^{\otimes k}$ with norm (largest singular value) at most 1 . The smallest $k$ for which $H$ admits such a decomposition is called the locality of $H$. The terms are normalized such that $\left\|H_{i}\right\| \leq 1$ for all $i$. A family of Hamiltonians $\left\{H_{i}\right\}$ acting on increasing numbers of qubits is called local if all $H_{i}$ are $k$-local for some $k$ independent of $n$ (for us $k$ will always be 2).

The local Hamiltonian problem is the prototypical QMA-complete problem, as 3SAT is for NP.

Definition 2.1. Let $k \geq 2$ be an integer. The $k$-local Hamiltonian problem is to decide, given a family of $k$-local Hamiltonians $\left\{H_{n}\right\}_{n \in \mathbb{N}}$ such that $H_{n}$ acts on $n$ qubits, and functions $a, b: \mathbb{N} \rightarrow$ $(0,1)$ such that $b-a=\Omega\left(\right.$ poly $\left.^{-1}(n)\right)$, if the smallest eigenvalue of $H_{n}$ is less than $a(n)$ or greater than $b(n)$.

Here we restrict our attention to Hamiltonians

$$
H=\frac{1}{m} \sum_{i=1}^{m} H_{i}
$$

for which each term $H_{i}$ can be written as a linear combination of tensor products of Pauli $I, \sigma_{X}$ and $\sigma_{Z}$ observables only. Such Hamiltonians are known to be QMA complete for some constant $k$ (see Lemma 22 of [15] for a proof).

\subsection{State-Dependent Distance Measure and Approximations}

We make extensive use of a state-dependent distance between measurements that has been frequently used in the context of entangledprover interactive proof systems (see e.g. $[14,15]$ ). For $\rho$ a positive semidefinite matrix and $X$ any linear operator define

$$
\operatorname{Tr}_{\rho}(X)=\operatorname{Tr}(\rho X) .
$$

For any two operators $S, T$, define the state-dependent distance between $S$ an $T$ on a $\rho$ as

$$
\mathrm{d}_{\rho}(S, T):=\sqrt{\operatorname{Tr}_{\rho}\left((S-T)^{\dagger}(S-T)\right)} .
$$

Based on the state-dependent distance we define a distance between POVMs, given by summing the state-dependent distance between the square roots of the POVM elements. Let $\left\{M^{a}\right\}$ and $\left\{N^{a}\right\}$ be two POVMs with the same number of outcomes, indexed by $a$, and let $\rho$ be a quantum state. Then the state-dependent distance between the POVMs $M$ and $N$ on $\rho$ is denoted as $\mathrm{d}_{\rho}(\sqrt{M}, \sqrt{N})$ and defined as

$$
\mathrm{d}_{\rho}(\sqrt{M}, \sqrt{N})=\left(\sum_{a} \mathrm{~d}_{\rho}\left(\sqrt{M^{a}}, \sqrt{N^{a}}\right)^{2}\right)^{1 / 2} .
$$

While this notation is ambiguous (since the sum over outcomes is not explicitly indicated), context will always make it clear which notion of $\mathrm{d}_{\rho}$ is intended. We will also drop the square roots in the case of POVMs that are projective measurements.

To simplify the notation, let $A^{a}=\sqrt{M^{a}}$ and $B^{a}=\sqrt{N^{a}}$. Then this distance can be rewritten as:

$$
\begin{aligned}
\mathrm{d}_{\rho}(\sqrt{M}, \sqrt{N})^{2} & =\sum_{a} \operatorname{Tr}_{\rho}\left(\left(A^{a}-B^{a}\right)^{2}\right) \\
& =2-2 \sum_{a} \operatorname{Re} \operatorname{Tr}_{\rho}\left(A^{a} B^{a}\right),
\end{aligned}
$$

where we used the fact that $A^{a}$ and $B^{a}$ are Hermitian and their squares sum to identity. If we specialize to the case of projective measurements with binary outcomes, we get the following relations (here $A=A^{1}-A^{-1}$ and $B=B^{1}-B^{-1}$ are the observables associated to the measurements):

$$
\begin{aligned}
\mathrm{d}_{\rho}(\sqrt{M}, \sqrt{N})^{2} & =2-\operatorname{Tr}_{\rho}\left(A^{1} B^{1}+A^{-1} B^{-1}+B^{1} A^{1}+B^{-1} A^{-1}\right) \\
& =\frac{1}{2} \operatorname{Tr}_{\rho}\left((A-B)^{2}\right) \\
& =\mathrm{d}_{\rho}(A, B)^{2} .
\end{aligned}
$$

This distance measure has the following useful property:

LEMMA 2.2. Let $\rho$ be positive semidefinite, $C$ be a linear operator such that $\left\|C C^{\dagger}\right\| \leq K$ and $S, T$ linear operators. Then

$$
\left|\operatorname{Tr}_{\rho}(C S)-\operatorname{Tr}_{\rho}(C T)\right| \leq \sqrt{2 K} \mathrm{~d}_{\rho}(S, T) .
$$

Likewise, if $\left\{C_{a}\right\}$ a family of operators with $\left\|\sum_{a} C_{a} C_{a}^{\dagger}\right\| \leq K$ and $\left\{M^{a}\right\}$ and $\left\{N^{a}\right\}$ POVMs. Then

$$
\left|\sum_{a} \operatorname{Tr}_{\rho}\left(C_{a} \sqrt{M^{a}}\right)-\sum_{a} \operatorname{Tr}_{\rho}\left(C_{a} \sqrt{N^{a}}\right)\right| \leq \sqrt{K} \mathrm{~d}_{\rho}(\sqrt{M}, \sqrt{N}) .
$$

The proof of both results is an application of the Cauchy-Schwarz inequality; we refer to [24] for a proof.

A second measure of proximity that is often convenient is the consistency. As before, let $\left\{M^{a}\right\}$ and $\left\{N^{a}\right\}$ be POVMs with the same number of outcomes. Then their consistency is defined as

$$
\mathrm{C}_{\rho}(M, N)=\operatorname{Re}\left(\sum_{a} \operatorname{Tr}_{\rho}\left(M^{a} N^{a}\right)\right)
$$

so that by (3) we have

$$
\mathrm{d}_{\rho}(\sqrt{M}, \sqrt{N})^{2}=2-2 \mathrm{C}_{\rho}(\sqrt{M}, \sqrt{N}) .
$$

For collections of binary observables $\{A(a)\}$ and $\{B(a)\}$ we use

$$
\mathrm{C}_{\rho}(A, B)=\frac{1}{2} \operatorname{Re}\left(1+\sum_{a} \operatorname{Tr}_{\rho}(A(a) B(a))\right) .
$$

A useful property of the consistency is that if $M$ and $N$ are POVMs acting on two separate subsystems of $\rho$, applying Naimark dilation to each of them results in projective measurements $M^{\prime}$ and $N^{\prime}$ and a state $\rho^{\prime}$ such that $\mathrm{C}_{\rho}(M, N)=\mathrm{C}_{\rho^{\prime}}\left(M^{\prime}, N^{\prime}\right)$. 
Given two observables $A$ and $B$, the product $A B$ is an observable if and only if $A$ and $B$ commute. The following lemma shows how to define a "product" observable $C$ when $A$ and $B$ commute only approximately in state-dependent distance, such that the action of $C$ on the state is close to $A B$ (and $B A$ ).

Lemma 2.3. Let $\rho$ be a density matrix and $A, B$ observables such that $\mathrm{d}_{\rho}(A B, B A) \leq \delta$ for some $\delta \geq 0$. Let $C$ be the observable defined by

$$
C=\frac{A B+B A}{|A B+B A|}
$$

where we use the convention that $M /|M|$ is defined as the identity on the kernel of $M$. Then

$$
\max \left\{\mathrm{d}_{\rho}(C, A B), \mathrm{d}_{\rho}(C, B A)\right\} \leq \frac{\sqrt{2}}{2} \delta .
$$

We refer to [24] for the proof.

Our calculations will often require estimates of the averaged squared distance of the form $\mathrm{E}_{x} \mathrm{~d}_{\rho}\left(A_{x}, B_{x}\right)^{2}=O(\epsilon)$ where the expectation is taken according to some distribution on $x$ (always over a finite set) that will be clear from context. We introduce the following notation to represent the same estimate:

$$
A_{x}|\psi\rangle \approx_{\epsilon}^{x} B_{x}|\psi\rangle .
$$

Here $|\psi\rangle$ can be understood as any purification of $\rho$, with the usual convention that operators are extended to act as identity on spaces on which they are not defined. If the symbol $x$ is omitted then the distribution should be clear from context. If it needs to be specified we may write e.g. $A_{x}|\psi\rangle \approx_{\epsilon}^{x \mid x_{1}=0} B_{x}|\psi\rangle$, meaning that the distribution on $x$ is the one clear from context (typically, uniform on $\left.\{0,1\}^{n}\right)$, conditioned on the first bit of $x$ being a 0 . Although the notation can be ambiguous when taken out of context we hope that it will help make some of the more cumbersome derivations more transparent.

\subsection{Nonlocal Games}

In the paper we formulate a number of tests meant to be executed between a verifier and $r$ players (sometimes also called provers), for $r \geq 1$ an integer. These tests all take the form of a classical one-round interaction: the verifier samples an $r$-tuple of questions and sends one question to each player; the players each provide an answer to the verifier, who decides to accept or reject. If the verifier accepts the players are said to win the game.

We call a tuple $(N,|\psi\rangle)$, where $|\psi\rangle \in \mathcal{H}_{1} \otimes \cdots \otimes \mathcal{H}_{r}$ is an entangled state on the joint space of all $r$ players, and $N$ a collection of POVM for each player and possible question to the player, a strategy for the players in $G$. Note that we may always assume $|\psi\rangle$ is a pure state and all POVM are projective.

Given a game $G$ we denote by $\omega^{*}(G)$ the highest probability of winning that can be achieved by $r$ players sharing quantum entanglement. For a more thorough introduction to nonlocal games in a similar framework as used here we refer to e.g. [15].

One of our tests uses nonlocal games as a means to enforce anticommutation relations between a player's observables. Towards this we introduce the following definition.
Definition 2.4 (Anticommutation game). Let $\omega_{\mathrm{ac}}^{*} \in(0,1]$ and $\delta:[0,1] \rightarrow[0,1]$ a continuous function such that $\delta(0)=0$. A two-player game $G$ is called a $\left(\omega_{\mathrm{ac}}^{*}, \delta\right)$ anticommutation game if $\omega^{*}(G) \geq \omega_{\mathrm{ac}}^{*}$ and moreover there exists questions $q_{X}, q_{Z}$ (called special questions) to the second player and $\{ \pm 1\}$-valued functions $f_{X}, f_{Z}$ defined on the player's set of possible answers to questions $q_{X}, q_{Z}$ respectively such that the following two properties hold:

(1) Completeness: There exists a strategy using the shared state $|\mathrm{EPR}\rangle{ }_{A B}^{\otimes m}$ for some $m \geq 1$ and projective measurements that achieves the optimal success probability $\omega_{\mathrm{ac}}^{*}$, and is such that measurement operators $\left\{A_{q}^{a}\right\} \in \operatorname{Pos}\left(\mathcal{H}_{A}\right)$ for the second player satisfy $\sum_{a} f_{X}(a) A_{q_{X}}^{a}=\sigma_{X} \otimes \mathbb{I}$ and $\sum_{a} f_{Z}(a) A_{q_{Z}}^{a}=\sigma_{Z} \otimes \mathbb{I}$, where $\sigma_{X}$ and $\sigma_{Z}$ act on the first EPR pair and the identity on the remaining EPR pairs. Moreover, for every question $q$ received by the second player and answer $a$, the projector $A_{q}^{a}$ can be written as $A_{q}^{a}=\sum_{j} \Pi_{j}$ where each $\Pi_{j}$ is the projector onto an eigenspace of a tensor product of $\sigma_{X}, \sigma_{Z}$ and $\mathbb{I} .^{3}$ We call such a strategy an honest strategy for $G$.

(2) Soundness: Let a projective strategy for the players in $G$ be given such that the strategy uses entangled state $|\psi\rangle \in$ $\mathcal{H}_{A} \otimes \mathcal{H}_{B}$ and measurement operators $\left\{A_{q}^{a}\right\} \in \operatorname{Pos}\left(\mathcal{H}_{A}\right)$ for the second player. Then for any $\epsilon>0$, provided the strategy has success probability at least $\omega_{\mathrm{ac}}^{*}-\epsilon$ in $G$, there exists isometries $U: \mathcal{H}_{A} \rightarrow \mathbb{C}^{2} \otimes \mathcal{H}_{A^{\prime}}$ and $V: \mathcal{H}_{B} \rightarrow \mathbb{C}^{2} \otimes \mathcal{H}_{B^{\prime}}$ and a state $\left|\psi^{\prime}\right\rangle \in \mathcal{H}_{A^{\prime}} \otimes \mathcal{H}_{B^{\prime}}$ such that if

$$
\begin{gathered}
X=\sum_{a} f_{X}(a) A_{q_{X}}^{a} \text { and } Z=\sum_{a} f_{Z}(a) A_{q_{Z}}^{a} \\
\text { then } \| U \otimes V|\psi\rangle-|\mathrm{EPR}\rangle \otimes\left|\psi^{\prime}\right\rangle \| \leq \delta \text { and } \\
\max \left\{\mathrm{d}_{\rho}\left(X, U^{\dagger}\left(\sigma_{X} \otimes \mathbb{I}_{A^{\prime}}\right) U\right),\right. \\
\left.\mathrm{d}_{\rho}\left(Z, V^{\dagger}\left(\sigma_{Z} \otimes \mathbb{I}_{B^{\prime}}\right) V\right)\right\} \leq \delta
\end{gathered}
$$

The CHSH game [4] and the Magic Square game [21, 27] are both known to be anti-commutation games. For the former, see e.g. [20] and for the latter, [7,32]. The advantage of the CHSH game is that there is an optimal strategy which only requires a single EPR pair of entanglement. The Magic Square has the advantage of having value 1 , but an optimal strategy requires two EPR pairs.

Lemma 2.5. The CHSH game is a $\left(\cos ^{2} \pi / 8, O(\sqrt{\epsilon})\right)$ anticommutation game. The Magic Square game is a $(1, O(\sqrt{\epsilon}))$ anticommutation game.

\section{THE LINEARITY TEST}

We state and analyze a variant of the classic 3-query linearity test of Blum, Luby, and Rubinfeld [2] (BLR) that can be played with two entangled players. The two-player test is based on the idea of oracularization with a dummy question introduced in [13]. Our analysis builds on [14], who analyze a 3-player variant. Their proof is an extension of the Fourier-analytic proof due to Håstad to the matrix-valued setting. We analyze the two-player variant using similar techniques.

\footnotetext{
${ }^{3}$ This seemingly ad-hoc condition is needed for the use of the anticommutation game in the Hamiltonian self-test described in Section 5, but not in the Pauli braiding test from Section 4.
} 
We note that the use of two players, rather than three as in the original test, is essential for our applications to self-testing. Ultimately we will require the provers to succeed in a linearity test performed in either of two mutually incompatible bases (e.g. the $X$ and $Z$ bases). Two provers can achieve this by sharing a maximally entangled state, but there is no tripartite state that would allow three entangled provers to obtain consistent answers whenever they measure their share of the state in either the $X$ or the $Z$ basis. (Formulated differently, $\sigma_{X} \otimes \sigma_{X}$ and $\sigma_{Z} \otimes \sigma_{Z}$ share a common +1 eigenvector, the EPR pair; $\sigma_{X} \otimes \sigma_{X} \otimes \sigma_{X}$ and $\sigma_{Z} \otimes \sigma_{Z} \otimes \sigma_{Z}$ do not. This is a manifestation of entanglement monogamy.)

We show the result in two steps. First we show that any set of quantum observables satisfying linearity relations approximately in expectation can be "rounded" to a nearby set of observables satisfying these relations exactly.

Theorem 3.1. Suppose there exist observables $\{A(a)\}_{a \in\{0,1\}^{n}}$ in $\operatorname{Obs}(\mathcal{H})$ acting on a state $\rho \in \mathrm{D}(\mathcal{H})$ such that

$$
\mathbf{E}_{a, b} \operatorname{Tr}_{\rho}(A(a) A(b) A(a+b)) \geq 1-\delta .
$$

Then there exists an extended state $\rho^{\prime}=\rho \otimes \mid$ anc $\rangle\langle$ anc $| \in \mathrm{D}\left(\mathcal{H} \otimes \mathcal{H}^{\prime}\right)$ and observables $\{\mathcal{A}(a)\}$ in $\operatorname{Obs}\left(\mathcal{H} \otimes \mathcal{H}^{\prime}\right)$ such that

$$
\begin{aligned}
& \mathcal{A}(a) \mathcal{A}(b)=\mathcal{A}(a+b) \quad \forall a, b \in\{0,1\}^{n} \\
& \mathrm{E}_{a} \mathrm{~d}_{\rho^{\prime}}(\mathcal{A}(a), A(a))^{2} \leq \delta
\end{aligned}
$$

Here, and throughout this paper, the notation $a+b$ denotes the bitwise XOR of $a$ and $b$, i.e. the sum of $a$ and $b$ viewed as elements of the additive group $\mathbb{Z}_{2}^{n}$. We call observables $\{\mathcal{A}(a)\}$ satisfying the first set of relations in (8) exactly linear.

Proof. For every $u \in\{0,1\}^{n}$ consider the Fourier transform $\hat{A}^{u}=\mathrm{E}_{a}(-1)^{a \cdot u} A(a)$. Define measurement operators $B^{u}=\left(\hat{A}^{u}\right)^{2}$. By Parseval's identity, these operators form a POVM. By Naimark's theorem, there exists an ancilla space $\mathcal{H}^{\prime}, \mid$ anc $\rangle\langle$ anc $| \in \mathrm{D}\left(\mathcal{H}^{\prime}\right)$, and a projective measurement $\left\{C^{u}\right\}$ on $\mathcal{H} \otimes \mathcal{H}^{\prime}$ that simulates $\left\{B^{u}\right\}$. Introduce observables

$$
\mathcal{A}(a)=\sum_{u}(-1)^{u \cdot a} C^{u} .
$$

From the orthogonality of the projectors $C^{u}$ it follows that for all $a, b, \mathcal{A}(a) \mathcal{A}(b)=\mathcal{A}(a+b)$. Write

$$
\begin{aligned}
\mathrm{E}_{a} & \mathrm{C}_{\rho^{\prime}}(A(a), \mathcal{A}(a)) \\
& =\frac{1}{2}+\frac{1}{2} \mathrm{E}_{a} \operatorname{Re} \operatorname{Tr}_{\rho^{\prime}}(A(a) \mathcal{A}(a)) \\
& =\frac{1}{2}+\frac{1}{2} \mathrm{E}_{a} \operatorname{Re}\left(\sum_{u} \operatorname{Tr}_{\rho}\left((-1)^{u \cdot a} A(a)\left(\hat{A}^{u}\right)^{2}\right)\right) \\
& =\frac{1}{2}+\frac{1}{2} \sum_{u} \operatorname{Tr}_{\rho}\left(\left(\hat{A}^{u}\right)^{3}\right) .
\end{aligned}
$$

To conclude, note that

$$
\sum_{u} \operatorname{Tr}_{\rho}\left(\left(\hat{A}^{u}\right)^{3}\right)=\mathbf{E}_{a b} \operatorname{Tr}_{\rho}(A(a) A(b) A(a+b)),
$$

and use the assumption made in the theorem and the relation between $\mathrm{C}_{\rho^{\prime}}$ and $\mathrm{d}_{\rho^{\prime}}^{2}$.
The verifier performs the following one-round interaction with two players. He starts by choosing one of the players at random and labels her Alice; the other player is labeled Bob. In each test each player is sent a pair of $n$-bit strings. The $n$-bit strings are always assumed to be sent in lexicographic order.

(1) Choose two strings $a, b \in\{0,1\}^{n}$ uniformly at random. Send $(a, b)$ to Alice.

(2) Let $c$ be with equal probability either $a, b$, or $a+b$, and let $c^{\prime} \in\{0,1\}^{n}$ be chosen uniformly at random. Send $\left(c, c^{\prime}\right)$ to Bob.

(3) The players reply with $\alpha, \beta \in\{ \pm 1\}$ and $\gamma, \gamma^{\prime} \in\{ \pm 1\}$ respectively. Depending on the value of $c$ the verifier performs one of the following two tests:

(a) Consistency test: if $c=a$ (resp. $b$ ), accept if and only if both players return the same value as their answer to that question: $\gamma=\alpha$ (resp. $\gamma=\beta$ ).

(b) Linearity test: if $c=a+b$, accept if and only if $\gamma=\alpha \beta$.

\section{Figure 1: The two-player linearity test}

Next we exhibit a two-player game such that any strategy which succeeds with probability at least $1-\epsilon$ in the game must satisfy the assumption (7) of Theorem 3.1 for some $\delta=O(\sqrt{\epsilon})$.

Theorem 3.2. Suppose two players Alice and Bob succeed in the linearity test of Figure 1 with probability at least $1-\epsilon$, using $a$ shared state $|\psi\rangle_{A B} \in \mathcal{H}_{A} \otimes \mathcal{H}_{B}$ and projective measurements $\left\{M_{a, b}^{\alpha, \beta}\right\}_{\alpha, \beta} \in$ $\operatorname{Pos}\left(\mathcal{H}_{A}\right)$ and $\left\{N_{a, b}^{\alpha, \beta}\right\}_{\alpha, \beta} \in \operatorname{Pos}\left(\mathcal{H}_{B}\right)$ respectively. Consider the POVM $\left\{\tilde{M}_{a}^{\alpha}\right\}_{\alpha}$ whose elements are given by $\tilde{M}_{a}^{\alpha}:=\mathrm{E}_{b} \sum_{\beta} M_{a, b}^{\alpha, \beta}$, and let $\left\{A_{a}^{\alpha}\right\}_{\alpha} \in \operatorname{Pos}\left(\mathcal{H}_{A} \otimes \mathcal{H}_{A^{\prime}}\right)$ be the projective measurement obtained by Naimark dilation of $\tilde{M}$.

Then the observables $A(a):=A_{a}^{0}-A_{a}^{1}$ satisfy

$$
\mathbf{E}_{a, b} \operatorname{Tr}_{\rho^{\prime}}(A(a) A(b) A(a+b))=1-O(\sqrt{\epsilon}),
$$

where $\rho^{\prime}=|\psi\rangle\langle\psi|\otimes|$ anc $\rangle\left\langle\right.$ anc $\left.\right|_{\mathcal{H}_{\mathrm{A}^{\prime}}}$.

Proof of Theorem 3.2. Introduce the following measurement operator on $\mathcal{H}_{B}$,

$$
N_{a \mid a b}^{\alpha}=\sum_{\beta} N_{a b}^{\alpha \beta} .
$$

Note that for every $a, b$ and $\alpha, N_{a \mid a b}^{\alpha}$ is a projector since we assumed each $M_{a b}^{\alpha \beta}$ is as well. Suppose that the players' acceptance probability conditioned on the verifier performing the consistency part of the test (i.e. $c=a$ or $c=b$ ) is $1-\epsilon_{c}$, while conditioned on the verifier performing the linearity part of the test (i.e. $c=a+b$ ) it is $1-\epsilon_{l}$, so that $\epsilon=2 \epsilon_{c} / 3+\epsilon_{l} / 3$. Let $\rho=|\psi\rangle\left\langle\left.\psi\right|_{A B}\right.$. By definition of the consistency test,

$$
1-\epsilon_{c}=\mathrm{E}_{a b} \mathrm{C}_{\rho}\left(\tilde{M}_{a}, N_{a \mid a b}\right) .
$$

Using Naimark's dilation theorem there is an ancilla space $\mathcal{H}_{A^{\prime}}$ and $\mid$ anc $\rangle\langle$ anc $| \in \mathrm{D}\left(\mathcal{H}_{\mathrm{A}}^{\prime}\right)$ such that the POVM $\left\{\tilde{M}_{a}^{\alpha}\right\}$ acting on $\mathcal{H}_{A}$ can be simulated by a projective measurement $\left\{A_{a}^{\alpha}\right\}$ acting on 
$\rho^{\prime}=\rho \otimes|\operatorname{anc}\rangle\left\langle\left.\operatorname{anc}\right|_{\mathcal{H}_{\mathrm{A}^{\prime}}}\right.$. Let $d(a \mid a b)=\mathrm{d}_{\rho^{\prime}}\left(A_{a}, N_{a \mid a b}\right)$, so that by Jensen's inequality, (5) and (9),

$$
\begin{aligned}
\mathbf{E}_{a b} d(a \mid a b) & \leq \sqrt{\mathbf{E}_{a b} d(a \mid a b)^{2}} \\
& =O\left(\sqrt{1-\mathbf{E}_{a b} \mathrm{C}_{\rho}\left(\tilde{M}_{a}, N_{a \mid a b}\right)}\right) \\
& =O\left(\sqrt{\epsilon_{c}}\right) .
\end{aligned}
$$

Now compute

$$
\begin{aligned}
\mathbf{E}_{a b} & \operatorname{Tr}_{\rho^{\prime}}(A(a) A(b) A(a+b)) \\
= & \mathbf{E}_{a b} \sum_{\alpha \beta} \operatorname{Tr}_{\rho^{\prime}}\left(A_{a}^{\alpha} A_{b}^{\beta} A_{a+b}^{\alpha \beta}-A_{a}^{\alpha} A_{b}^{\beta} A_{a+b}^{-\alpha \beta}\right) \\
= & 2 \mathbf{E}_{a b} \sum_{\alpha \beta} \operatorname{Tr}_{\rho^{\prime}}\left(A_{a}^{\alpha} A_{b}^{\beta} A_{a+b}^{\alpha \beta}\right)-1 \\
\geq & 2 \mathbf{E}_{a b}\left(\sum_{\alpha \beta} \operatorname{Tr}_{\rho^{\prime}}\left(A_{a+b}^{\alpha \beta} N_{a \mid a b}^{\alpha} N_{b \mid a b}^{\beta}\right)\right. \\
& -O(d(a \mid a b)+d(b \mid a b))) \\
& -1 \\
= & 1-O\left(\epsilon_{l}+\sqrt{\epsilon_{c}}\right),
\end{aligned}
$$

where the inequality uses Lemma 2.2 and the last line is by (10) and, by definition of the linearity test,

$$
\begin{aligned}
1-\epsilon_{l} & =\mathrm{E}_{a b} \sum_{\alpha, \beta} \operatorname{Tr}_{\rho}\left(\tilde{M}_{a+b}^{(\alpha \beta)} N_{a b}^{\alpha \beta}\right) \\
& =\mathrm{E}_{a b} \sum_{\alpha, \beta} \operatorname{Tr}_{\rho}\left(\tilde{M}_{a+b}^{(\alpha \beta)} N_{a \mid a b}^{\alpha} N_{b \mid a b}^{\beta}\right),
\end{aligned}
$$

since the POVM elements $N_{a \mid b}^{\alpha \beta}$ are projectors.

\section{THE PAULI BRAIDING TEST}

In this section we combine the linearity test with an anticommutation test based on any anticommutation game $G_{\mathrm{ac}}$ satisfying Definition 2.4 to devise a two-player test for which the honest strategy consists of applying tensor products of single-qubit observables in the set $\left\{\sigma_{X}(a) \sigma_{Z}(b), a, b \in\{0,1\}\right\}$. We show that for any strategy with near-optimal success probability there exists a (local) isometry under which the players' observables are close (in the state-dependent distance) on average to operators satisfying the Pauli commutation and anti-commutation ("braiding") relations perfectly.

\subsection{The Protocol}

The protocol for the Pauli braiding test is described in Figure 2. In the protocol there are several possible types of queries that each player may receive. For convenience we give them the following names:

(1) A $W$-query, represented by $(W, a, b)$, where $W \in\{X, Z\}$ and $a, b$ are uniformly random strings in $\{0,1\}^{n}$. The expected answer is two bits $\alpha, \beta \in\{-1,1\}$.

(2) A G-query, represented by $(q, a, b)$ where $q$ is a question in $G_{\mathrm{ac}}$ and $a, b$ are uniformly random strings in $\{0,1\}^{n}$. The
Let $G_{\text {ac }}$ be a two-player anticommutation game, with special questions $q_{X}, q_{Z}$. The verifier performs the following one-round interaction with two players. He starts by choosing one of the players at random and labels them Alice; the other player is labeled Bob. In each test a player will be sent a label and a pair of $n$-bit strings. The $n$-bit strings are always assumed to be sent in lexicographic order.

(1) Linearity test: The verifier chooses a basis setting $W \in$ $\{X, Z\}$ and sends it to both players. He executes the twoplayer linearity test with the players.

(2) Anticommutation test: The verifier chooses two strings $a, b \in\{0,1\}^{n}$ such that $a \cdot b=1 \bmod 2$ uniformly at random, and sends $(a, b)$ to both players. He executes the game $G_{\mathrm{ac}}$ with the players and accepts if and only if they succeed.

(3) Consistency test: The verifier chooses two strings $a, b \in$ $\{0,1\}^{n}$ such that $a \cdot b=1 \bmod 2$ uniformly at random, and a basis setting $W \in\{X, Z\}$. He sends $(W, a, b)$ to Alice. With probability $1 / 2$ each,

- He samples a question $q$ from the second player's distribution in $G_{\mathrm{ac}}$ and sends $(q, a, b)$ to Bob. If $q=q_{X}$ (resp. $q=q_{Z}$ ) he accepts if and only if Alice's answer associated to $a$ (resp. $b$ ) equals $f_{W}(\alpha)$, where $\alpha$ is Bob's answer and $f_{W}$ the function from Definition 2.4. Otherwise, he accepts automatically.

- He selects a uniformly random $c \in\{0,1\}^{n}$ and sends $(W, a, c)$ to Bob. He accepts if and only if the product of Alice and Bob's answers associated to the query string $a$ is +1 .

\section{Figure 2: The two-player Pauli braiding test}

expected answer is a single value $\alpha$ taken from the answer alphabet in $G$.

To each query is associated an intended behavior of the player, which is specified as part of the honest strategy given in the following definition.

Definition 4.1. The honest strategy for the two players in the Pauli braiding test consists of the following. Let $U, V$ be unitaries to an optimal strategy in $G_{\mathrm{ac}}$ as in Definition 2.4, and recall that by the completeness property this strategy can be implemented by sharing $m$ EPR pairs of entanglement.

The players share the state $|\psi\rangle_{A B}=|\mathrm{EPR}\rangle_{A B}^{\otimes n} \otimes|\mathrm{EPR}\rangle_{A^{\prime} B^{\prime}}^{\otimes(m-1)}$. Upon receiving a query, a player performs the following depending on the type of the query:

- $W$-query $(W, a, b)$, for $W \in\{X, Z\}$ : measure the compatible observables $\sigma_{W}(a)$ and $\sigma_{W}(b)$ on its share of $|\mathrm{EPR}\rangle \underset{A B}{\otimes n}$, and return the two outcomes.

- $G$-query $(q, a, b)$. Suppose the query is sent to Alice, the case of Bob being treated symmetrically. Let $W_{a, b}: \mathbb{C}^{2^{n}} \rightarrow$ $\mathbb{C}^{2^{n}}$ be a unitary such that $W_{a, b} \sigma_{X}(a) W_{a, b}^{\dagger}=\mathbb{I}_{\mathbb{C}^{2 n-1}} \otimes \sigma_{X}$ and $W_{a, b} \sigma_{Z}(b) W_{a, b}^{\dagger}=\mathbb{I}_{\mathbb{C}^{2 n-1}} \otimes \sigma_{Z}$. (Such a $W_{a, b}$ exists and can be agreed upon by the players since in a $G$-query it is 
always the case that $a \cdot b=1 \bmod 2$, and both players are sent the same pair $(a, b)$.) Let $\left\{A_{q}^{\alpha}\right\}_{\alpha}$ be the projective measurement on $\mathbb{C}^{2} \otimes \mathcal{H}_{A^{\prime}}$ associated with the first player in a honest strategy in $G$. Then Alice performs the projective measurement

$$
\left\{\left(W_{a, b}^{\dagger} \otimes \mathbb{I}_{A^{\prime}}\right)\left(\mathbb{I}_{\mathbb{C}^{2 n-1}} \otimes A_{q}^{\alpha}\right)\left(W_{a, b} \otimes \mathbb{I}_{A^{\prime}}\right)\right\}_{\alpha}
$$

and returns the outcome.

Having defined the honest strategy for the players we introduce some notation associated with arbitrary strategies in the protocol. We specify a strategy using the shorthand $\left(N,|\psi\rangle_{A B}\right)$. Here $|\psi\rangle_{A B}$ denotes the bipartite state shared by the players, and $N$ the collection of POVM that the players apply in response to the different types of queries they can be asked. Using Naimark's theorem we may assume without loss of generality that $|\psi\rangle_{A B}$ is a pure state and each player's POVM is projective.

Given a query $(X, a, b)$ (resp. $(Z, a, b))$, we denote the projective measurement that is applied by Alice (resp. Bob) by $\left\{N_{a b}^{\alpha \beta}\right\}_{\alpha, \beta}$ (resp. $\left.\left\{M_{a b}^{\alpha \beta}\right\}_{\alpha, \beta}\right)$. Since the protocol treats the players symmetrically we may assume that these operators are the same for both Alice and Bob (see e.g. [31, Lemma 2.5]). By taking appropriate marginals over the answers we define associated observables for the players, $X^{A}(a)$ and $Z^{A}(b)$ for the first player and $X^{B}(a)$ and $Z^{B}(b)$ for the second, as

$$
\begin{aligned}
& X^{A}(a)=\frac{1}{2^{n}} \sum_{b \in\{0,1\}^{n}} \sum_{\beta \in\{ \pm 1\}}\left(N_{a b}^{1 \beta}-N_{a b}^{-1 \beta}\right), \\
& Z^{A}(b)=\frac{1}{2^{n}} \sum_{a \in\{0,1\}^{n}} \sum_{\alpha \in\{ \pm 1\}}\left(M_{a b}^{\alpha 1}-M_{a, b}^{\alpha-1}\right) .
\end{aligned}
$$

Observables $X^{B}(a)$ and $Z^{B}(b)$ for the second player are defined similarly.

Finally we use $X^{\prime A}(a, b)$ and $Z^{\prime A}(a, b)$ to denote the observables defined via (6) from Alice's strategy upon questions $\left(q_{X}, a, b\right)$ and $\left(q_{Z}, a, b\right)$ respectively.

\subsection{Statement of Results}

We state the analysis of the Pauli braiding test in two parts: first we show that success in the test implies that observables (11) constructed from Alice and Bob's measurement operators approximately obey certain relations; then we show that these relations imply the existence of a local isometry under which the operators are close to operators satisfying the relations exactly.

Theorem 4.2. Suppose a strategy $\left(N,|\psi\rangle_{A B}\right)$ succeeds in the Pauli braiding test (Figure 2) with probability at least $\omega_{\text {pauli }}^{*}-\epsilon$, when the game $G_{\mathrm{ac}}$ is an $\left(\omega_{\mathrm{ac}}^{*}, \delta\right)$ anticommutation game. Then the following approximate relations hold, where operators $W^{D}$ are defined in (11) for $W \in\{X, Z\}$ and $D \in\{A, B\}$ and $\rho=|\psi\rangle\langle\psi|$.

(1) (Approximate consistency) For $W \in\{X, Z\}$,

$$
\mathbf{E}_{a} \mathrm{~d}_{\rho}\left(W^{A}(a), W^{B}(a)\right)^{2}=O(\epsilon) ;
$$

(2) (Approximate linearity) For $W \in\{X, Z\}$,

$$
\mathbf{E}_{a, b} \mathrm{~d}_{\rho}\left(W^{A}(a) W^{A}(b), W^{A}(a+b)\right)^{2}=O(\sqrt{\epsilon}) ;
$$

(3) (Approximate anticommutation)

$\mathrm{E}_{a, b \mid a \cdot b=1} \mathrm{~d}_{\rho}\left(X^{A}(a) Z^{A}(b),-Z^{A}(b) X^{A}(a)\right)^{2}=O(\sqrt{\epsilon}+\delta(\epsilon)) ;$

(4) (Approximate commutation)

$\mathbf{E}_{a, b \mid a \cdot b=0} \mathrm{~d}_{\rho}\left(X^{A}(a) Z^{A}(b), Z^{A}(b) X^{A}(a)\right)^{2}=O\left(\epsilon^{\frac{1}{4}}+\sqrt{\delta(\epsilon)}\right)$.

We note that the constant $\omega_{\text {pauli }}^{*}$ is given by

$$
\omega_{\text {pauli }}^{*}=\frac{2}{3}+\frac{1}{3} \omega_{\mathrm{ac}}^{*}
$$

where $\omega_{\mathrm{ac}}^{*} \in(0,1]$ is the winning parameter associated with the $\left(\omega_{\mathrm{ac}}^{*}, \delta\right)$ anticommutation game $G_{\mathrm{ac}}$ used in the protocol. Thus if $\omega_{\mathrm{ac}}^{*}=1$ then $\omega_{\text {pauli }}^{*}=1$ as well.

Theorem 4.3. Suppose given a bipartite state $|\psi\rangle_{A B} \in \mathcal{H}_{A} \otimes$ $\mathcal{H}_{B}$, and observables $\left\{X^{A}(a)\right\}_{a \in\{0,1\}^{n}},\left\{Z^{A}(b)\right\}_{b \in\{0,1\}^{n}}$ on $\mathcal{H}_{A}$ and

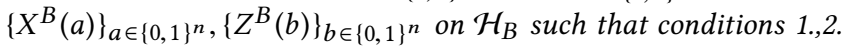
and 3. in Theorem 4.2 are satisfied, for some $\epsilon>0$ and $\delta(\epsilon)=O(\sqrt{\epsilon}) .{ }^{4}$ Then there exists a state

$$
\begin{aligned}
|\Psi\rangle_{A B} & =|\psi\rangle_{A B} \otimes|\mathrm{EPR}\rangle_{A^{\prime} A^{\prime \prime}} \otimes|\mathrm{EPR}\rangle_{B^{\prime} B^{\prime \prime}} \\
& \in\left(\mathcal{H}_{A} \otimes\left(\mathbb{C}_{A^{\prime}}^{2} \otimes \mathbb{C}_{A^{\prime \prime}}^{2}\right)^{\otimes n}\right) \otimes\left(\mathcal{H}_{B} \otimes\left(\mathbb{C}_{B^{\prime}}^{2} \otimes \mathbb{C}_{B^{\prime \prime}}^{2}\right)^{\otimes n}\right)
\end{aligned}
$$

and observables $\left\{P^{A}(a, b)\right\}$ on $A A^{\prime} A^{\prime \prime}$ such that, if $\rho=|\Psi\rangle\langle\Psi|$,

(a) (Approximate consistency)

$$
\mathbf{E}_{a} \mathrm{~d}_{\rho}\left(P^{A}(a, 0), X^{A}(a) \otimes \mathbb{I}_{A^{\prime} A^{\prime \prime}}\right)^{2}=O\left(\epsilon^{1 / 8}\right)
$$

and

$$
\mathbf{E}_{b} \mathrm{~d}_{\rho}\left(P^{A}(0, b), Z^{A}(b) \otimes \mathbb{I}_{A^{\prime} A^{\prime \prime}}\right)^{2}=O\left(\epsilon^{1 / 8}\right) .
$$

(b) (Pauli braiding) For all $a, b, a^{\prime}, b^{\prime} \in\{0,1\}^{n}$,

$$
P^{A}(a, b) P^{A}\left(a^{\prime}, b^{\prime}\right)=(-1)^{a^{\prime} \cdot b} P^{A}\left(a+a^{\prime}, b+b^{\prime}\right) .
$$

Likewise, there exist observables $\left\{P^{B}(a, b)\right\}$ on $B B^{\prime} B^{\prime \prime}$ satisfying analogous relations.

We note that the Pauli braiding relations expressed in (b) imply the existence of an isomorphism such that the operators $P^{A}(a, b)$ (resp. $\left.P^{B}(a, b)\right)$ are mapped to "true" Pauli operators $\sigma_{X}^{A}(a) \sigma_{Z}^{A}(b)$ (resp. $\left.\sigma_{X}^{B}(a) \sigma_{Z}^{B}(b)\right)$.

Before moving to the proofs we state an immediate, but powerful, application of the theorems to the problem of establishing dimension witnesses. For this it is sufficient to note the following well-known fact:

FACT 4.4. Let $\rho$ be a density matrix on $\mathbb{C}^{\otimes n} \otimes \mathbb{C}^{\otimes n}$ and $\epsilon>0$ such that

$$
\frac{1}{2^{n}} \sum_{P \in\{X, Z\}^{n}} \operatorname{Tr}\left(\left(\sigma_{P} \otimes \sigma_{P}\right) \rho\right) \geq 1-\epsilon,
$$

where $\sigma_{P}=\sigma_{P_{1}} \otimes \cdots \otimes \sigma_{P_{n}}$. Then

$$
\left\langle\left.\mathrm{EPR}\right|^{\otimes n} \rho \mid \mathrm{EPR}\right\rangle^{\otimes n} \geq 1-\epsilon .
$$

Proof. Observe that

$$
|\mathrm{EPR}\rangle\langle\mathrm{EPR}| \geq \frac{1}{2}\left(\sigma_{X} \otimes \sigma_{X}+\sigma_{Z} \otimes \sigma_{Z}\right)
$$

\footnotetext{
${ }^{4}$ The restriction on $\delta$ is not necessary, but it is satisfied for both the CHSH and Magic Square games, and simplifies the presentation.
} 
Combining this fact and Theorems 4.2 and 4.3 gives the following consequence: a robust self-test for $n$ EPR pairs.

Corollary 4.5. Suppose given a strategy $\left(N,|\psi\rangle_{A B}\right)$ for the players in the Pauli braiding test (Figure 2) with success probability $\omega_{\text {pauli }}^{*}-\epsilon$, for some $\epsilon>0$. Then there exists a local isometry $\Phi=$ $\left(\Phi^{A}: \mathcal{H}_{A} \rightarrow \mathcal{H}_{A^{\prime}} \otimes \mathcal{H}_{A^{\prime \prime}}, \Phi^{B}: \mathcal{H}_{B} \rightarrow \mathcal{H}_{B^{\prime}} \otimes \mathcal{H}_{B^{\prime \prime}}\right)$ such that

$$
\begin{gathered}
\operatorname{Tr}\left(\left(\langle \mathrm { EPR } | _ { A ^ { \prime } B ^ { \prime } } ^ { \otimes n } \otimes \mathbb { I } _ { A ^ { \prime \prime } B ^ { \prime \prime } } ) \left(\Phi^{A} \otimes \Phi^{B}\left(|\psi\rangle\left\langle\left.\psi\right|_{A B}\right)\right)\right.\right.\right. \\
\left.\left(|\mathrm{EPR}\rangle_{A^{\prime} B^{\prime}}^{\otimes n} \otimes \mathbb{I}_{A^{\prime \prime} B^{\prime \prime}}\right)\right)=1-O\left(\epsilon^{1 / 8}\right) .
\end{gathered}
$$

By instantiating the anticommutation game $G_{\text {ac }}$ used in the test with the Magic Square game we obtain a robust self-test for $n$ EPR pairs in which the optimal strategy only requires the use of $(n+1)$ EPR pairs and is accepted with probability $1 .^{5}$

\subsection{Proof of Theorem 4.3}

The poof of Theorem 4.2 follows along the lines of previous works such as [15] and is omitted. Here we a sketch of the proof of Theorem 4.3. Detailed proofs of both theorems can be found in the full version of this paper [24].

Proof of Theorem 4.3 (Sketch). Adjoin two $n$-qubit registers $A^{\prime}$ and $A^{\prime \prime}$ to Alice's system, and initialize them in the state $|\mathrm{EPR}\rangle_{A^{\prime} A^{\prime \prime}}^{\otimes n}$. Define new observables $X^{\prime}(a):=X^{A}(a) \otimes \sigma_{X}(a)_{A^{\prime}} \otimes \mathbb{I}_{A^{\prime \prime}}$ and $Z^{\prime}(b):=Z^{A}(b) \otimes \sigma_{Z}(b)_{A^{\prime}} \otimes \mathbb{I}_{A^{\prime \prime}}$. Further define observables

$$
C(a, b):=\frac{X^{\prime}(a) Z^{\prime}(b)+Z^{\prime}(b) X^{\prime}(a)}{\left|X^{\prime}(a) Z^{\prime}(b)+Z^{\prime}(b) X^{\prime}(a)\right|},
$$

where the notation $|\cdot|$ denotes the matrix absolute value and we use the convention $0 / 0=1$. It follows from the assumptions made in the theorem (i.e. properties 1,2 and 3 in Theorem 4.2) that $C(a, b)$ satisfies approximate linearity over $\mathbb{Z}_{2}^{2 n}$, i.e. that

$$
C(a, b) C\left(a^{\prime}, b^{\prime}\right)|\Psi\rangle \approx^{a, b, a^{\prime}, b^{\prime}} C\left(a+a^{\prime}, b+b^{\prime}\right)|\Psi\rangle .
$$

Applying Theorem 3.1 (over $\{0,1\}^{2 n}$ ), we conclude that there exist observables $D(a, b)$ acting on an extension of Alice's system by an ancilla state, satisfying $D(a, b) D\left(a^{\prime}, b^{\prime}\right)=D\left(a+a^{\prime}, b+b^{\prime}\right)$ and $\mathbf{E}_{a, b} \mathrm{~d}_{\rho}(D(a, b), C(a, b))^{2}=O\left(\epsilon^{1 / 8}\right)$. Set

$$
P^{A}(a, b):=D(a, b) \otimes \sigma_{X}(a)_{A^{\prime \prime}} \sigma_{Z}(b)_{A^{\prime \prime}} .
$$

Using the exact linearity of $D(a, b)$ and the approximate consistency between $D(a, b)$ and $C(a, b)$, it can be verified that $P^{A}(a, b)$ satisfies the desired properties in the conclusion of the theorem (approximate consistency and Pauli braiding).

\section{THE HAMILTONIAN SELF-TEST}

In this section, we build on the Pauli braiding test to construct a test that distinguishes between the cases when a Hamiltonian given as input has ground state energy below, or higher than, pre-specified thresholds (i.e. in the former case the players will have a strategy with high success probability in the protocol, whereas in the latter any strategy will have low success probability). Due to the nature of our tests we restrict attention to $n$-qubit Hamiltonians specified by

${ }^{5}$ In fact, for the case of the Magic Square game it is not hard to see that there always exists an optimal strategy in the test using $\max (2, n)$ EPR pairs. a linear combination of $m$ terms, each of which is a tensor product of single-qubit $I, \sigma_{X}$ or $\sigma_{Z}$ Pauli operators.

Recall the Pauli braiding test analyzed in the previous section. As we saw (Corollary 4.5) this test can be used as a robust self-test for an $n$-qubit maximally entangled state. In order to test non-maximally entangled states, we proceed as in $[11,15]$ by requiring the (honest) players to share a qubit-by-qubit encoding of the ground state of the Hamiltonian, where each qubit is encoded using a simple $r$-qubit CSS code. As elucidated in [15], any code state, thought of as a bipartite entangled state across any one of its qubits and the others, is maximally entangled. This allows us to lift the two-player tests which constitute the Pauli braiding test to $r$-player tests, where each player holds one qubit ("share") of the encoding of each qubit of the ground state, and one of the players (to be called the special player) plays the role of Alice while the remaining $(r-1)$ players (to be called the composite player) play the role of Bob.

The essential property of the constituent tests of the Pauli braiding test that permit this lifting is that all of the measurements performed by Bob in the honest strategy can be implemented by measuring the tensor product of Pauli operators $\sigma_{X}, \sigma_{Z}$, and II on a state of $n$ EPR pairs. (For the anticommutation test, this is ensured by the completeness condition in Definition 2.4, and for the other tests, it can be seen to hold for both Alice and Bob's measurements). These operators can be implemented transversally in any CSS code, and moreover in a way such that marginal distribution of queries received by the special player and each of the composite players is identical, as we show below.

\subsection{The Protocol}

We describe the protocol in detail. The input is an $n$-qubit local Hamiltonian $H$ that can be expressed as

$$
H=\frac{1}{m} \sum_{\ell=1}^{m} H_{\ell}, \quad H_{\ell}=\alpha_{\ell} \sigma_{X}\left(a_{\ell}\right) \sigma_{Z}\left(b_{\ell}\right),
$$

for $\alpha_{\ell} \in[-1,1]$ and $a_{\ell}, b_{\ell} \in\{0,1\}^{n}$ such that $a_{\ell} \wedge b_{\ell}=0^{n}$ for all $\ell \in\{1, \ldots, m\}$. The verifier interacts with $r$ players, where $r$ is the number of qubits of codewords in the CSS code chosen for the protocol (such as Steane's 7-qubit code, as described in Section 2.2, in which case $r=7$ ).

Although the protocol is to be performed with $r$ "physical" players, part of the protocol consists in applying the Pauli braiding test, which is formulated as a two-player test in the previous section. To translate between the $r$ players and the two players in the Pauli braiding test we introduce two "logical" players. A query to the logical players (as specified in the Pauli braiding test) is mapped to a query to the $r$ physical players as follows. One of the physical players is chosen at random to play the role of the first logical player (Alice), called the special player. The remaining $(r-1)$ physical players together play the role of the second logical player (Bob), called the composite player. $^{6}$ For a given query $Q$ to the special player of a type among those specified in the Pauli braiding test we

\footnotetext{
${ }^{6}$ The physical players remain isolated throughout the protocol and are never allowed to communicate; it is only for purposes of analysis that we group $(r-1)$ physical players into a single logical player. In particular the physical players are never told which logical player they are associated with, and the distribution of queries to any physical player is the same whether it plays the role of the special or composite player.
} 
Given a local Hamiltonian $H=\sum_{\ell=1}^{m} \alpha_{\ell} H_{\ell}$, where $\alpha_{\ell} \in[-1,1]$ and each $H_{\ell}=\sigma_{X}\left(a_{\ell}\right) \sigma_{Z}\left(b_{\ell}\right)$. Let $p \in(0,1)$ be a parameter of the protocol.

The verifier performs one of the following three tests at random, the first with probability $(1-p)$ and the second and third with probability $p / 2$ each.

(1) (Pauli braiding test) Choose one of the $r$ players uniformly at random to be the special player. The other players form the composite player. Simulate the Pauli braiding test with these two players, where the role of Alice is assigned to the special player and the role of Bob to the composite player.

(2) (Energy test) Choose $\ell \in\{1, \ldots, m\}$ uniformly at random. Define an operator $Q_{\ell}$ acting on $r n$ qubits by replacing each $\sigma_{X}$ in $H_{\ell}$ with $X_{\text {logical }}$ on the $r$-qubit code state, and $\sigma_{Z}$ by $Z_{\text {logical }}$. Send each player a query $(X Z, a, b)$ representing the associated share of $Q_{\ell}$. The players should each return two values in $\{-1,1\}$.

The verifier takes the product of all values received. If its sign disagrees with that of $\alpha_{\ell}$, he accepts. If they agree, he rejects with probability $\left|\alpha_{\ell}\right|$ and accepts otherwise.

(3) (Energy consistency test) Choose one of the $r$ players uniformly at random to be the special player. The other players form the composite player. Let $W \in\{X, Z\}$, each chosen with probability $1 / 2$. Also choose $a, b$ according to the same distribution as in the energy test. The verifier performs one of the following tests, each chosen with the indicated probability.

- With probability $1 / 2$, send the special player $(X Z, a, b)$, and the composite player $\overline{(W, c, c+a)}$ if $W=X$ and $\overline{(W, c, c+b)}$ if $W=Z$, where $c \in\{0,1\}^{n}$ is chosen uniformly at random. Accept if the special player's answer agrees with the product of the composite player's two answers.

- With probability $1 / 4$, send the special player $(W, c, d)$, and the composite player $\overline{(W, c, c+a)}$, where $c, d \in$ $\{0,1\}^{n}$ are chosen uniformly at random. Accept if the special player and composite player agree on the answer associated with $c$.

- With probability $1 / 4$, send the special player $(W, c+$ $a, d)$, and the composite player $\overline{(W, c, c+a)}$, where $c, d \in\{0,1\}^{n}$ are chosen uniformly at random. Accept if the special player and composite player agree on the answer associated with $c+a$.

Figure 3: The Hamiltonian self-test

define a complementary query $\bar{Q}$ for the composite player as per the following lemma.

LemmA 5.1. For any $X$-query or $Z$-query, there exists a complementary query $\bar{Q}$ such that

(1) The query associated to each physical player forming the composite player in $\bar{Q}$ is of the same type as $Q$. In particular the distribution on query strings is as specified by the query type.

(2) If all players apply the honest strategy and provide answers $\alpha, \beta$ to $Q$ and $\bar{\alpha}, \bar{\beta}$ to $\bar{Q}$ respectively, where $\bar{\alpha}$ and $\bar{\beta}$ are each obtained as the product of the answer to the corresponding query coming from each of the physical players making up the composite player, it holds that $\alpha \bar{\alpha}=\beta \bar{\beta}=+1$.

Proof. Both items follow from the properties of CSS codes described in Section 2.2.

We can then define associated observables for the players, $\hat{X}(a)$ and $\hat{Z}(b)$ for the special player and $\bar{X}(a)$ and $\bar{Z}(b)$ for the composite player, exactly as in (11).

Aside from the Pauli braiding test, the protocol considers two other tests called the energy test and the energy consistency test. In the energy test, the verifier asks the players to measure a randomly chosen term in the Hamiltonian. The consistency test is needed to relate the operators applied in the energy test to those applied in the Pauli braiding test.

\subsection{Statement of Results}

Our main result regarding the Hamiltonian self-test is given in the following theorem, which states the completeness and soundness guarantees of the protocol described in Figure 3.

THEOREM 5.2. There exists a constant $0<d<1$ such that the following holds. Let $H$ be a (not necessarily local) Hamiltonian with $m$ terms over $n$ qubits of the form (13), and $\lambda_{\min }(H)$ the smallest eigenvalue of $H$. Then for every $\eta>0$ there is a choice $p=\Theta\left(\eta^{1-d}\right)$ for the probability of performing the energy test in Protocol 3 such that the maximum probability $\omega^{*}(H)$ with which any $r$-player strategy succeeds in the protocol satisfies

$$
\begin{aligned}
1- & \frac{p}{8}\left(\lambda_{\min }(H)+\frac{2}{m} \sum_{\ell=1}^{m}\left|\alpha_{l}\right|\right) \\
& \leq \omega^{*}(H) \leq 1-\frac{p}{8}\left(\lambda_{\min }(H)+\frac{2}{m} \sum_{\ell=1}^{m}\left|\alpha_{l}\right|\right)+\eta .
\end{aligned}
$$

Corollary 1.2 follows by applying Theorem 5.2 to the amplified Hamiltonian $H^{\prime}=\mathbb{I}^{\otimes a}-\left(\mathbb{I}-\left(H-a^{-1} \mathbb{I}\right)\right)^{\otimes a}$ over an qubits, for an appropriately chosen $a$ which is polynomial in $n$.

The proof of the theorem relies on the following two lemmas, together with the analysis of the Pauli braiding test given in Section 4.2. For details, consult the full version [24].

Lemma 5.3. Given a Hamiltonian $H$ as in (13), the acceptance probability of the energy test, when the correct Pauli operators are applied by each player on its respective register of the $(r n)$-qubit encoding of an $n$-qubit state $|\psi\rangle$, is

$$
\begin{aligned}
\omega_{\text {energy }}^{*}(H,|\psi\rangle) & =1-\left(\frac{1}{2 m} \sum_{\ell=1}^{m} \frac{\left|\alpha_{\ell}\right|+\alpha_{\ell}\left\langle\psi\left|H_{\ell}\right| \psi\right\rangle}{2}\right) \\
& =1-\left(\frac{1}{4}\langle\psi|H| \psi\rangle+\frac{1}{2 m} \sum_{\ell}\left|\alpha_{\ell}\right|\right),
\end{aligned}
$$

where $H_{\ell}=\sigma_{X}\left(a_{\ell}\right) \sigma_{Z}\left(b_{\ell}\right)$ is the $\ell$-th term in the Hamiltonian. 
LEMmA 5.4. Suppose the strategy $(N,|\psi\rangle)$ for the players succeeds in the energy consistency test and the Pauli braiding test with probability $1-\epsilon$ each. Then

$$
\frac{1}{m} \sum_{\ell=1}^{m} \|\left(\hat{H}_{\ell}-\hat{X}(a) \hat{Z}(b)\right)|\psi\rangle \|^{2}=O\left(\epsilon^{1 / 32}\right),
$$

where $a$ and $b$ are strings such that $H_{\ell}=\sigma_{X}(a) \sigma_{Z}(b)$, and $\hat{H}_{\ell}$ is the observable applied by the special player upon receiving the query $(X Z, a, b)$ in the energy test.

Moreover, the honest strategy succeeds in the test with probability 1.

\section{ACKNOWLEDGMENTS}

AN was supported by NSF Grant CCF-1629809 and ARO Contract Number W911NF-12-0486. TV was supported by NSF CAREER Grant CCF-1553477 and AFOSR YIP award number FA9550-161-0495. Parts of this work was completed while the first author was visiting the Institute for Quantum Information and Matter (IQIM) at the California Institute of Technology, and while both authors were visiting the Perimeter Institute in Waterloo, ON. Both authors acknowledge funding provided by the IQIM, an NSF Physics Frontiers Center (NSF Grant PHY-1125565) with support of the Gordon and Betty Moore Foundation (GBMF-12500028).

\section{REFERENCES}

[1] Dorit Aharonov, Itai Arad, and Thomas Vidick. 2013. The Quantum PCP Conjecture. Technical Report. arXiv:1309.7495. Appeared as guest column in ACM SIGACT News archive Volume 44 Issue 2, June 2013, Pages 47-79.

[2] Manuel Blum, Michael Luby, and Ronitt Rubinfeld. 1993. Self-testing/correcting with applications to numerical problems. F. Comput. System Sci. 47 (1993), 549595.

[3] A. R. Calderbank and Peter W. Shor. 1996. Good quantum error-correcting codes exist. Phys. Rev. A 54 (1996), 1098-1105. Issue 2. arXiv:quant-ph/9512032.

[4] John F. Clauser, Michael A. Horne, Abner Shimony, and Richard A. Holt. 1969. Proposed Experiment to Test Local Hidden-Variable Theories. Phys. Rev. Lett. 23 (Oct 1969), 880-884. Issue 15. DOI : https://doi.org/10.1103/PhysRevLett.23.880

[5] Richard Cleve, Peter Høyer, Ben Toner, and John Watrous. 2004. Consequences and Limits of Nonlocal Strategies. (2004). arXiv:quant-ph/0404076.

[6] Andrea W. Coladangelo. 2016. Parallel self-testing of (tilted) EPR pairs via copies of (tilted) CHSH. Technical Report. arXiv:1609.03687.

[7] Matthew Coudron and Anand Natarajan. 2016. The parallel-repeated Magic Square game is rigid. Technical Report. arXiv:1609.06306.

[8] Toby Cubitt and Ashley Montanaro. 2014. Complexity classification of local Hamiltonian problems. In Foundations of Computer Science (FOCS), 2014 IEEE 55th Annual Symposium on. IEEE, 120-129.

[9] Irit Dinur. 2007. The PCP theorem by gap amplification. f. ACM 54, 3, Article 12 (June 2007). DOI : https://doi.org/10.1145/1236457.1236459

[10] Joseph Fitzsimons and Michal Hajdušek. 2015. Post hoc verificatin of quantum computing. Technical Report. arXiv:1512.04375.
[11] Joseph Fitzsimons and Thomas Vidick. 2015. A multiprover interactive proof system for the local Hamiltonian problem. In Proceedings of the 2015 Conference on Innovations in Theoretical Computer Science. ACM, 103-112.

[12] Daniel Gottesman. 1997. Stabilizer Codes and Quantum Error Correction. (1997). arXiv:quant-ph/9705052 arXiv:quant-ph/9705052.

[13] Tsuyoshi Ito, Hirotada Kobayashi, and Keiji Matsumoto. 2009. Oracularization and Two-Prover One-Round Interactive Proofs against Nonlocal Strategies. In Proceedings: Twenty-Fourth Annual IEEE Conference on Computational Complexity (CCC 2009). 217-228.

[14] Tsuyoshi Ito and Thomas Vidick. 2012. A multi-prover interactive proof for NEXP sound against entangled provers. Proc. 53rd FOCS (2012), 243-252.

[15] Zhengfeng Ji. 2016. Classical verification of quantum proofs. In Proceedings of the 48th Annual ACM SIGACT Symposium on Theory of Computing. ACM, 885-898.

[16] Zhengfeng Ji. 2016. Compression of Quantum Multi-Prover Interactive Proofs. Technical Report. arXiv:1610.03133.

[17] Hirotada Kobayashi and Keiji Matsumoto. 2003. Quantum Multi-Prover Interactive Proof Systems with Limited Prior Entanglement. FCSS 66 (2003), 429-450. Issue 3. arXiv:cs/0102013.

[18] Matthew McKague. 2014. Self-testing graph states. In Theory of Quantum Computation, Communication, and Cryptography. Springer, 104-120.

[19] Matthew McKague. 2015. Self-testing in parallel. (2015). arXiv:1511.04194 arXiv:1511.04194.

[20] M McKague, T H Yang, and V Scarani. 2012. Robust self-testing of the singlet. Journal of Physics A: Mathematical and Theoretical 45, 45 (2012), 455304. http: //stacks.iop.org/1751-8121/45/i=45/a=455304

[21] N David Mermin. 1990. Simple unified form for the major no-hidden-variables theorems. Physical Review Letters 65, 27 (1990), 3373.

[22] Carl A. Miller and Yaoyun Shi. 2014. Robust Protocols for Securely Expanding Randomness and Distributing Keys Using Untrusted Quantum Devices. In Proceedings of the 46th Annual ACM Symposium on Theory of Computing (STOC '14). ACM, New York, NY, USA, 417-426. DOI : https://doi.org/10.1145/2591796. 2591843

[23] Ashley Montanaro and Ronald de Wolf. 2013. A survey of quantum property testing. arXiv preprint arXiv:1310.2035 (2013).

[24] Anand Natarajan and Thomas Vidick. 2016. Robust self-testing of many-qubit states. Technical Report. arXiv:1610.03574.

[25] Michael A. Nielsen and Isaac L. Chuang. 2001. Quantum Computation and Quantum Information. Cambridge University Press.

[26] Dimiter Ostrev and Thomas Vidick. 2016. Entanglement of approximate quantum strategies in XOR games. Technical Report. arXiv:1609.01652.

[27] Asher Peres. 1990. Incompatible results of quantum measurements. Physics Letters A 151, 3-4 (1990), 107-108.

[28] Ben Reichardt, Falk Unger, and Umesh Vazirani. 2013. A classical leash for a quantum system: Command of quantum systems via rigidity of $\mathrm{CHSH}$ games. Nature 496, 7446 (2013), 456-460.

[29] Andrew Steane. 1996. Multiple-particle interference and quantum error correction. In Proceedings of the Royal Society of London A: Mathematical, Physical and Engineering Sciences, Vol. 452. The Royal Society, 2551-2577. arXiv:quant$\mathrm{ph} / 9601029$.

[30] Umesh Vazirani and Thomas Vidick. 2014. Fully Device-Independent Quantum Key Distribution. Phys. Rev. Lett. 113 (Sep 2014), 140501. Issue 14. DOI : https: //doi.org/10.1103/PhysRevLett.113.140501

[31] Thomas Vidick. 2013. Three-player entangled XOR games are NP-hard to approximate. In Proc. 54th FOCS.

[32] Xingyao Wu, Jean-Daniel Bancal, Matthew McKague, and Valerio Scarani. 2016. Device-independent parallel self-testing of two singlets. Physical Review A 93, 6 (2016), 062121 